\title{
Understanding Catalyst Stability Through Aberration-corrected STEM
}

\author{
A.R. Lupini, G.M. Veith, N.J. Dudney, and S. J. Pennycook \\ Materials Science and Technology Division, Oak Ridge National Laboratory. Oak \\ Ridge, TN 37831, U.S.A.
}

Catalysts play an essential role in energy production as well as reducing the pollution and energy consumption of many industrial processes. Characterizing and understanding how such catalysts work is one of the main challenges in producing more efficient, cost-effective and stable materials. Aberration-corrected scanning transmission electron microscopy (STEM) is uniquely well suited to studies of supported metal nanoparticle catalysts because of the Z-contrast imaging mode, where the intensity is approximately proportional to the square of the atomic number $Z$. Thus even single heavy atoms can be detected and quantities such as particle thickness as well as distribution can be measured [1,2]. A further advantage of aberrationcorrection, which is used to improve the resolution of the microscope, is that the depth of field is correspondingly decreased, allowing three-dimensional information to be extracted [3]. We have recently developed a magnetron sputtering technique, able to produce a variety of catalyst materials, which allow us to investigate the factors that affect the stability of supported nanoparticle catalysts. We have produced metal nanoparticles on a range of different and industrially relevant materials including, alumina, silica, titania, and other oxides, and are able to use the same method irrespective of the surface isoelectric point [4-6]. This combination of novel growth techniques and aberration-corrected STEM appears able to provide an unprecedented view into the growth and stability of supported nanoparticle catalysts.

Supported gold nanoparticles form a very interesting family of catalysts, since the size of the nanoparticles strongly affects the reaction rate for some useful processes $[1,7]$. However, the reaction rates produced by these materials are notoriously varied due to the large number of variables that influence the properties [8]. Using the magnetron sputtering technique and the sensitivity provided by aberration-corrected STEM we were able to probe some of these variables and uncover the important role that surface hydroxyl groups play in the stability of supported metal catalysts [6].

Gold nanoparticles grown on silica were found to be very stable, even when heated to relatively high temperatures [5]. Gold catalysts grown by sputtering on fumed titania were found to be unstable, with particles that were initially in the 2-3 nm size range agglomerating into large $\sim 20 \mathrm{~nm}$ particles over a few days at ambient conditions (Figure 1). Pre-treating the titania support material with either alkali or acid solutions, designed to mimic the conditions encountered during solution based deposition processes, results in a dramatic improvement in both the particle stability and the reaction rate. We did not find evidence that these changes were due to chlorine species, oxygen vacancies or an electronic effect. Instead we found that the stability and activity enhancements were due to the formation of hydroxyl species on the titania surface. Single gold atoms are visible on the $\mathrm{pH} 10$ treated titania (see Figure 1). Thus the $\mathrm{pH}$ changes during the conventional deposition-precipitation techniques result in a substantial modification of the support surface properties, which seems to be important in the formation of stable catalysts [6]. 


\section{References}

[1] A.A. Herzing et al, Science 321 (2008) 1331.

[2] S.N. Rashkeev, et al, Physical Review B 76 (2007) 035438.

[3] A.Y. Borisevich, et al, Proc. Natl. Acad. Sci. 103(2006) 3044.

[4] G.M. Veith, et al, Journal of Catalysis 231(2005) 151.

[5] G.M. Veith, et al, Journal of Catalysis 262 (2009) 92.

[6] G.M. Veith, A.R. Lupini and N.J. Dudney, J. Phys. Chem. 113 (2009) 269.

[7] M. Haruta, N. Yamada, H. Kobayashi, S.J. Iijima, Catal. 115 (1989) 301.

[8] F. Moreau, G.C. Bond, and A.O. Taylor, J. Catal. 231 (2005) 105.

[9] Research at ORNL was sponsored by the Division of Materials Sciences and Engineering of the US Department of Energy.
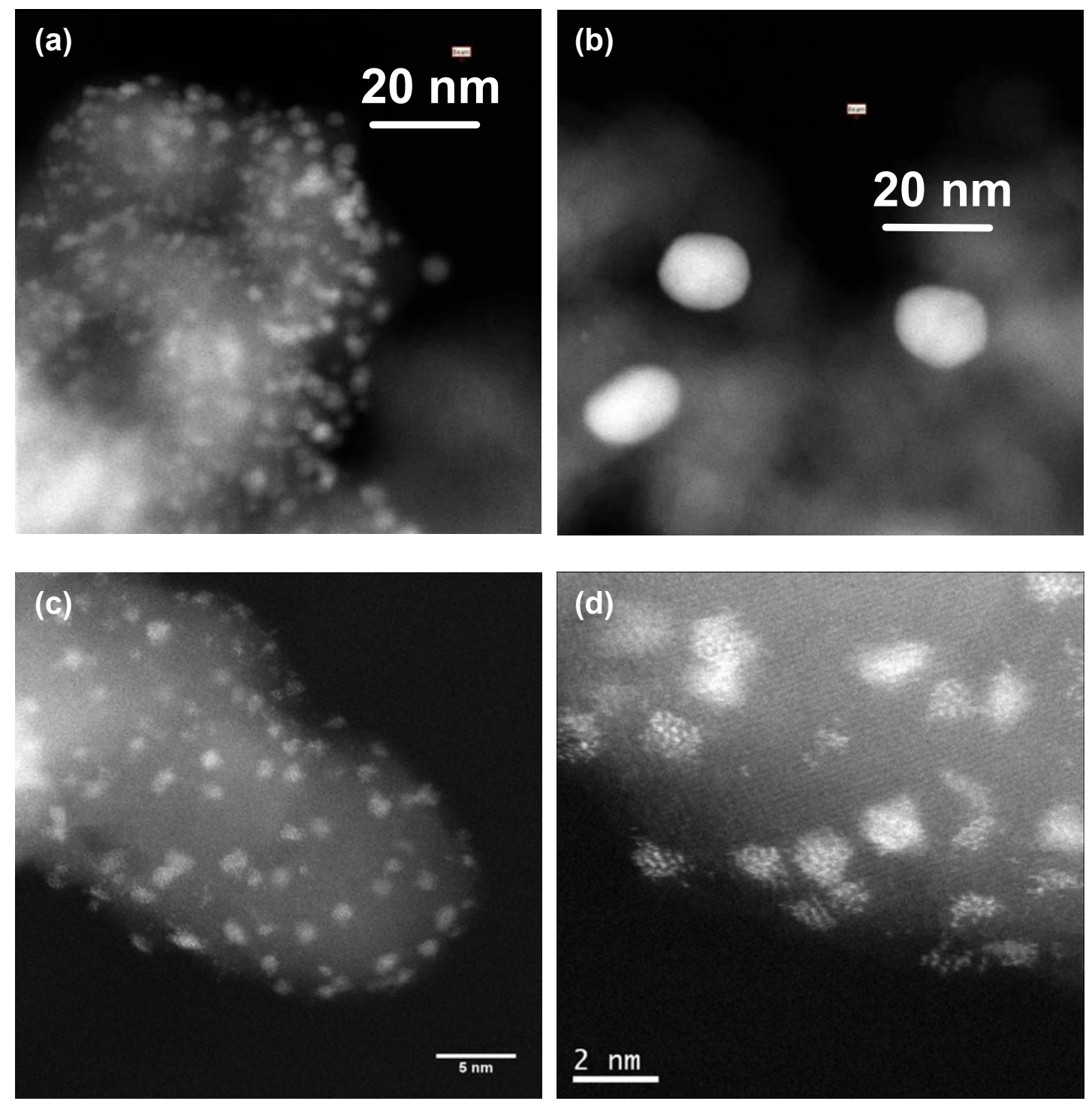

Figure 1. Z-contrast STEM images of gold on titania catalysts. Gold nanoparticles show up as bright white spots. (a) 2 hour old Au on natural Degussa P25. (b) 6 day old Au particles supported on natural Degussa P25. (c) 2 hour old Au on pH 10 treated Degussa P25. (d) 2 hour old Au on pH 10 treated Degussa P25. Some single Au atoms are visible on the $\mathrm{pH} 10$ treated sample. Adapted from reference [6]. 